\title{
Hugo Herrera
}

\section{Die Suche nach einem formal-gerechten Weltfrieden - Das Recht der Völker von John Rawls}

Obwohl sich im internationalen Bereich einige Elemente als permanent erweisen, stellen die Globalisierung und die letzten Kriege die politisch-philosophische Reflexion vor Fragen und eröffnen Debatten, die zu großen Teil neu sind. John Rawls ${ }^{1}$, einer der wichtigsten politischen Philosophen der angelsächsischen Welt, veröffentlichte drei Jahre vor seinem Tod Das Recht der Völker², ein Buch, in dem er Grundsätze einer internationalen politischen und juristischen Ordnung vorschlägt, die zu einem gerechten Frieden führen sollten.

\section{Die Vertragslebre von 1971}

Die völkerrechtliche Theorie Rawls' folgt zu bedeutenden Teilen seiner Vertragslehre von 19713. Ihr Ausgangspunkt ist ein fiktiver Urzustand, in dem gewisse Bedingungen gesetzt werden mit dem Zweck, Entscheidungen bzw. Regeln mit einem bestimmten Inhalt zu ermöglichen ${ }^{4}$. Die Annahme dieses Urzustandes ist Voraussetzung für gerechtes Handeln. Die Etablierung einer gerechten Ordnung hängt von zwei Prämissen dieses Urzustandes ab: Zum einen die Existenz von Marktbedingungen und Ungleichheit zwischen den Individuen. Rawls erkennt die Rolle des individuellen Interesses an. Zum anderen ein so genannter "Schleier des Nichtwissens « (veil of ignorance), der die konkrete Position der Individuen in der Gesell-

1 Rawls (Baltimore 1921-Lexington 2002) studierte in Cornell und Princeton und war von 1943 an für drei Jahre im pazifischen Raum als Soldat stationiert. Ab 1953 lehrte er an der Technischen Hochschule von Massachusetts (MIT) und später an der Universität Harvard. Rawls war verantwortlich für die Wiederbelebung der politischen Philosophie in der angelsächsischen Welt. Statt des dort herrschenden Utilitarismus vertrat er eine kantisch inspirierte politische Philosophie. Trotz der Nähe gibt es wichtige Unterschiede zwischen Rawls und Kant, von denen einige hier gezeigt werden.

2 Berlin/New York 2002 (1999), im Folgenden: RdV. 1993 veröffentlichte Rawls eine erste Version seiner völkerrechtlichen Theorie (in: S. Shute/S. Hurley [Hg.], On Human Rights. The Oxford Amnesty Lectures. New York 1993), die mit einigen Änderungen in Das Recht der Völker aufgenommen wird.

3 Vgl. J. Rawls, Eine Theorie der Gerechtigkeit (im Folgenden: TdG). Frankfurt/M. 1975 (1971).

4 Er stimmt hier mit Hobbes, Kant und Rousseau überein: vgl. Th. Hobbes, Leviathan. Stuttgart 2000 (übersetzt v. J.-P. Mayer), Kap. 13, S. 117; I. Kant, Reflexionen zur Rechtsphilosophie, in: Akademieausgabe. Berlin u.a., 1905 ff., Bd. 19, S. 503 (7734), 504 (7740), 564 (7956); J.-J. Rousseau, Diskurs über die Ungleichbeit. Discours sur l' inégalité. Kritische Ausgabe (hrsg. v. H. Meier). Paderborn u.a., 52001, S. 47-9. 
schaft verbirgt ${ }^{5}$. Gegenüber diesen Voraussetzungen ist die Suche nach der Verbesserung der Lebensbedingungen derer, die sich in den schlimmsten Positionen befinden werden, die plausibelste Strategie für die Verteidigung des eigenen Interesses. Gemäß diesen Voraussetzungen werden die folgenden Gerechtigkeitsprinzipien angenommen: »1. Jedermann soll gleiches Recht auf das umfangreichste System gleicher Grundfreiheiten haben, das mit dem gleichen System für alle anderen verträglich ist. 2. Soziale und wirtschaftliche Ungleichheiten sind so zu gestalten, dass (a) vernünftigerweise zu erwarten ist, dass sie zu jedermanns Vorteil dienen, und (b) sie mit Positionen und Ämtern verbunden sind, die jedem offenstehen « ${ }^{6}$.

Der Urzustand wird im Recht der Völker als formale moralphilosophische Konstruktion dargestellt: Umfassende inhaltliche Vorstellungen des Guten werden außer Betracht gelassen. Diese könnten die Etablierung einer fairen Gesellschaftsordnung behindern; sie sind nicht konsensfähig, denn es gibt Meinungsverschiedenheiten über ihre Geltung. Wenn solche Konzeptionen des Guten außer Kraft gesetzt sind, ist es möglich, einen »übergreifenden Konsens« (overlapping consensus) zu schaffen, der keine eigenständige Moralkonzeption entwirft, sondern als Desiderat aller partikularen Konzeptionen konstituiert wird. Die Entscheidung kann auf diese Weise Allgemeinheit und Unparteilichkeit erreichen und als universal akzeptabel erscheinen?

\section{Erweiterung der Theorie}

Rawls versucht, seine kontraktualistische Gerechtigkeitstheorie auf die Beziehungen zwischen Völkern (peoples) anzuwenden ${ }^{8}$. Ch. Beitz und Th. Pogge haben von der Theorie von 1971 ausgehend - eine universelle Anwendung des Differenzprinzips vorgeschlagen'. In seiner völkerrechtlichen Theorie fügt Rawls hingegen einen zweiten Urzustand hinzu, in dem die Vertragspartner nicht Individuen, sondern Völker - und ihre Vertreter - sind. Er versucht, die kosmopolitische Anwendung seiner Theorie und die Probleme, die mit dieser Anwendung verknüpft sind, zu vermeiden ${ }^{10}$, obwohl der Versuch mit der ursprünglichen Theorie unvereinbar zu sein scheint ${ }^{11}$.

5 Vgl. TdG, S. 29, $159 \mathrm{ff}$.

6 TdG, S. 81; vgl. $\$ 15$ und 63.

7 Vgl. RdV, S. 34 f., 158; J. Rawls, Die Idee des politischen Liberalismus. Aufsätze 19781989, hrsg. v. W. Hinsch. Frankfurt/M. 1992, S. 287, 305, 307, 310.

8 Vgl. I. Kant, Die Metaphysik der Sitten. Akademieausgabe, Bd. 6, S. 344; Zum ewigen Frieden, passim (Akademieausgabe, Bd. 8).

9 Vgl. Ch. Beitz, "Justice and International Relations «, in: Ch. Beitz/M. Cohen/T. Scanlon / J. Simons (Hg.), International Ethics. Princeton 1985, S. 282-311; Political Theory and International Relations. Princeton 1979, S. 153-163; Th. Pogge, »An Egalitarian Law of Peoples", in: Philosophy and Publics Affairs 23 (1994).

10 Vgl. RdV, S. 9, 143 ff.

11 Vgl. K.-H. Nusser, »Kant, Rawls und die >Revolutionen des Friedens<. Kants Nähe zur realistischen Interpretationen der internationalen Beziehungen«, in: Zeitschrift für Politik 1997, S. $361 \mathrm{ff}$. 
Vertragssubjekte sind ebenfalls nicht die Staaten. Staaten werden ausgeschlossen, weil der Begriff vom Staat zwei Souveranitätsbefugnisse enthält, die einer achtbaren und liberalen Gerechtigkeitskonzeption widersprechen: i) das Recht, als Mittel staatlicher Politik Krieg zu führen, und ii) die Autonomie hinsichtlich der Art und Weise, wie die Regierung das eigene Volk behandelt. Rawls zieht unter moralischem Aspekt das Volk dem Staat vor. Man kann Völkern moralische Motivationen zuschreiben, was nach ihm bei Staaten nicht möglich ist. Ihr Handeln wird nicht »ausschließlich « durch die "Staatsräson « bestimmt. Wenn sie gerecht und liberal sind, beschränken sie ihre vitalen Interessen so, wie es das Vernünftige erfordert. Staaten hingegen handeln gemäß den Kriterien der Staatsräson und neigen dazu, das Rationale - d.h. die Wirksamkeit der Mittel - dem Vernünftigen - d.h. der »Moralität« der Mittel - vorzuziehen ${ }^{12}$.

Rawls trennt sich damit von einer alten Denktradition, für die der Staat moralisch sein kann und soll13 und für die der Staat - als Form politischen Handelns verstanden - das Volk als Volk gestaltet und es seinen Mitgliedern so erst ermöglicht, politisch zu handeln ${ }^{14}$. Das Volk erscheint in den Beschreibungen Ralws' als eine Art ideales Humanitätsbild. Die Behauptung, dass Völker ständig dazu bereit seien, sich gegenseitig anzuerkennen, widerspricht jedoch der geschichtlichen Erfahrung. Völker können ebenso wie Menschen gute, aber auch neutrale oder schlechte Beziehungen untereinander haben. Der Grund der Aggressivität und des Bösen liegt im Menschen selbst, wovon Volk und Staat Ausdrücke sind ${ }^{15}$.

Gemäß der Nähe oder Distanz zu den liberal-demokratischen Prinzipien und zu den Grundsätzen des Rechts der Völker, unterscheidet Rawls fünf Arten von »heimischen Gesellschaften «: demokratisch-liberale Völker, achtbare Völker, outlaw regimes, belastete Gesellschaften und woblwollende absolutistische Gesellschaften ${ }^{16}$. Liberal-demokratische Völker sind solche, die die liberal-demokratischen Prinzipien und die Grundsätze des Rechts der Völker beachten. Achtbare Völker sind nicht liberal-demokratisch, aber sie haben eine Konsultationshierarchie, durch die die Mitglieder der Körperschaften am sozialen Entscheidungsprozess teilhaben, und handeln gemäß den Grundsätzen des Rechts der Völker. Diese zwei Arten von Gesellschaften werden als »wohlgeordnete Gesellschaften « bezeichnet. Outlaw regimes sind solche, die das Recht der Völker oder die Menschenrechte nicht beachten. Die belasteten Gesellschaften sind zwar nicht aggressiv und respektieren die Grundsätze des Rechts der Völker, sind aber von Umständen betroffen, die sie daran hindern, wohlgeordnete Gesellschaften zu sein. Wohlwollende absolutistische

12 Vgl. RdV, S. 19 f., 28, 30-32, 39; vgl. J. Rawls, Politischer Liberalismus (im Folgenden: PL). Frankfurt/M. 1998, S. $120 \mathrm{ff}$.

13 Vgl. z. B. Platon, Politeia 412c-e, 427e ff.; Aristoteles, Politik, 1252a 1 ff., b 27-30; Nikomachische Ethik 1094b 5-11.

14 Vgl. Aristoteles, Politik 1253a 1 ff.; C. Schmitt, Der Wert des Staates und die Bedeutung des Einzelnen. Hellerau 1917, S. 106-107.

15 Vgl. H. Kuhn, Der Staat. Eine philosophische Darstellung. München 1967, S. 16 ff., 53 ff.; C. Schmitt, Der Begriff des Politischen. Berlin ${ }^{6} 1996$, S. 26 ff., 35.

16 Vgl. RdV, S. 2. 
Gesellschaften schließlich sind solche, in denen die Menschenrechte geachtet werden, die die Grundsätze des Rechts der Völker beachten, in denen aber den Mitgliedern eine Rolle bei der Entscheidungsfindung verweigert wird.

Rawls wendet die Bedingungen des Vertrags analog auf die liberal-demokratischen Völker an. Der Urzustand im außenpolitischen Fall ist eine hypothetische Situation, in der faire und vernünftige Bedingungen vorausgesetzt werden. Anhand dieser legen rationale Vertreter von freien Völkern die fairen Kooperationsbedingungen fest, unter denen sie als freie und gleiche Völker handeln werden. Der Schleier der Unwissenheit verhüllt umfassende Lehren vom Guten (die liberalen Gesellschaften gründen sich nicht auf umfassende Lehren). Ähnlich wie im Urzustand nach der Theorie von 1971 die Individuen ihre konkrete Position in der Gesellschaft nicht wissen, kennen in der völkerrechtlichen Theorie die Vertreter der Völker nicht die Elemente, die die Situation der vertretenen Einheiten bestimmen (Zahl und Eigenschaften der Bevölkerung, Weite und Eigenschaften des Territoriums) $)^{17}$.

Rawls nennt öffentliche Vernunft die Gesamtheit der Inhalte, die die liberalen Völker verbunden hält. Die liberalen Völker als Einheiten stützen sich nicht auf umfassende Lehren des Guten. Die Beziehungen zu und der Bund mit anderen liberalen Völkern entstehen auch nicht auf Grund umfassender Lehren. Ein liberales Volk gründet sich auf die Idee des (autonomen) politisch Vernünftigen, das sich auf Bürger als Bürger (aber z.B. nicht als Anhänger einer Religion bzw. als Mitglieder von anderen Gemeinschaften) bezieht und das mit den formalen Grundsätzen des Liberalismus übereinstimmt ${ }^{18}$. Die Kriterien der Vernünftigkeit werden nicht abgeleitet (wie die kantischen praktischen Ideen), sondern einzeln aufgezählt und inhaltlich charakterisiert ${ }^{19}$. Die Vernünftigkeit der Kriterien kann ihrerseits nicht im Rahmen des politischen Liberalismus bewiesen werden ${ }^{20}$.

Die Gesellschaft der liberalen Völker gründet sich auf öffentlich vernünftigen Grundsätzen, die sich auf Völker als Völker beziehen, d.h. auf Begriffe, die von verschiedenen Völkern geteilt werden können. Im »heimischen« Fall handelt es sich bei

17 Vgl. RdV, S. 36 f.

18 Vgl. RdV, S. 107.

19 Für jeden Gegenstandsbereich werden relevante Kriterien für die Bestimmung des Vernünftigen angegeben. »So lassen sich vernünftige Bürger dadurch charakterisieren, dass sie bereit sind, unter Gleichen faire Kooperationsbedingungen anzubieten, und dass sie die Bürden des Urteilens anerkennen. Auch wird von ihnen gesagt, dass sie ausschließlich vernünftige umfassende Lehren bejahen. Solche Lehren wiederum sind vernünftig, wenn sie die wesentlichen Elemente einer liberalen demokratischen Ordnung anerkennen und in kohärenter und konsequenter Weise eine vernünftige Rangordnung der verschiedenen Werte des Lebens (seien es nun religiöse oder nichtreligiöse Werte) bieten. Obwohl diese Lehren vergleichsweise stabil sein sollten, können sie sich im Lichte dessen weiterentwickeln, was im Laufe der Entwicklung ihrer Tradition als gute und hinreichende Gründe akzeptiert wurde. Es ist darüber hinaus ganz allgemein vernünftig, mit einer Vielfalt von Meinungen in politischen Urteilen zu rechnen und deshalb unvernünftig, alle Formen der Mehrheitsentscheidung abzulehnen. Ohne sie wäre eine liberale Demokratie unmöglich«; ebd. S. 107 f.; vgl. PL, S. 25 f., 120-139, 175.

Vgl. RdV, S. 108; TdG, S. 38 f. 
der öffentlichen Vernunft um die wesentlichen Verfassungsinhalte und die Angelegenheiten grundlegender Gerechtigkeit im Hinblick auf die eigene Regierung. Im außenpolitischen Fall geht es bei ihr um die Beziehungen zwischen den Völkern ${ }^{21}$.

Im Urzustand, unter dem Schleier der Unwissenheit, sollten nach Rawls die freien Völker acht Grundsätze anerkennen: 1) Völker sind frei und unabhängig, ihre Freiheit und Unabhängigkeit müssen von anderen Völker geachtet werden. 2) Völker müssen Verträge und eingegangene Verpflichtungen erfüllen. 3) Völker sind gleich und müssen an Übereinkünften, die sie binden sollen, beteiligt sein. 4) Völkern obliegt eine Pflicht der Nichteinmischung ${ }^{22}$. 5) Sie haben das Recht auf Selbstverteidigung, aber kein Recht, Kriege aus anderen Gründen zu führen. 6) Sie müssen die Menschenrechte achten. 7) Wenn sie Kriege führen, müssen sie bestimmte Einschränkungen beachten ${ }^{23}$. 8) Völker sind dazu verpflichtet, anderen Völkern zu helfen, wenn diese unter ungünstigen Bedingungen leben, welche verhindern, dass sie eine gerechte oder achtbare politische und soziale Ordnung etablieren ${ }^{24}$.

Der vierte Grundsatz wird von Rawls eingeschränkt: Die Nichteinmischung "muss offensichtlich qualifiziert werden, wenn es um outlaw regimes und um schwerwiegende Menschenrechtsverletzungen geht ${ }^{25}$. Ein solcher Grundsatz passt also zu einer Gesellschaft wohlgeordneter Völker, versagt aber in anderen Fällen ${ }^{26}$.

Mit diesen Grundsätzen sei es möglich, einen Frieden zwischen liberal-demokratischen Völkern zu schaffen. Er nennt ihn einen »Frieden aus den richtigen Gründen«. Wie bei Kant ist der Frieden bei Rawls etwas anderes als ein Waffenstillstand und auch nicht bloßes Ergebnis eines Kräftegleichgewichts ${ }^{27}$. Es gebe bestimmte Bedingungen, die die Existenz eines Friedens zwischen liberalen Völkern festigen würden sowie die Tatsache erklären könnten, warum sie nur in gerechtfertigten Fällen Krieg führten (Selbstverteidigung, Verteidigung von Alliierten, Verteidigung gegen schwerwiegende Verletzungen der Menschenrechte): Die Anerkennung von Grundrechten und Freiheiten; der Vorrang dieser Rechte, Freiheiten und Lebenschancen, insbesondere gegenüber den Forderungen des Gemeinwohls und gegenü-

21 Vgl. RdV, S. 62 f.

22 Vgl. I. Kant, Zum ewigen Frieden, aaO. (FN 8), S. 346.

23 Ebd. S. $346 \mathrm{f}$.

24 Vgl. RdV, S. 41.

25 RdV, S. 42.

26 Neben den gemeinsamen Grundsätzen, sind die kooperativen Organisationen zwischen den Völkern wichtig. Rawls nennt drei solche grundlegenden Organisationen: Eine, um die Fairness des Handels zwischen Völkern zu gewährleisten; eine zweite, die es Völkern ermöglicht, Kredite von einem kooperativen Bankensystem aufzunehmen; und eine dritte - vergleichbar den Vereinten Nationen -, die Rawls als eine Konföderation der Völker (nicht der Staaten) bezeichnet; vgl. RdV, S. 47 f.; I. Kant, Zum ewigen Frieden, aaO. (FN 8), S. 354 ff. (Kant spricht von Staaten).

27 RdV, S. 49; I. Kant, Zum ewigen Frieden, ebd., S. 343. Dieser Frieden wird mit Hilfe einer Analogie plausibel gemacht: So wie im heimischen Fall die Bürger, während sie erwachsen werden und sich an der liberalen Welt, in der sie leben, beteiligen, einen Gerechtigkeitssinn entwickeln können, so könnten auch die Völker einen Sinn für das Recht der Völker entwickeln, sofern sie gemäß diesem handeln und in ihm auf diese Weise »erzogen « werden; vgl. RdV, S. 49 f. 
ber perfektionistischen Werten ${ }^{28}$; die sichere Versorgung der Bürger mit den für den besonnenen und wirksamen Gebrauch ihrer Freiheiten notwendigen Grundgüter ${ }^{29}$.

Der demokratische Frieden sei geschichtlich bewiesen: Seit 1800 hätten »sicher etablierte liberale Gesellschaften nicht mehr gegeneinander gekämpft « ${ }^{30}$. Diese Behauptung ist allerdings nicht genügend begründet und ziemlich trügerisch. Sie verliert ihre Überzeugungskraft, wenn man erstens bedenkt, dass die demokratischen, »sicher etablierten liberalen Gesellschaften« erst seit Ende des zweiten Weltkriegs in größerer Zahl existieren. Vorher war die Wahrscheinlichkeit von Kriegen zwischen solchen Gesellschaften somit sehr gering. Zweitens gilt, dass diese wenigen (um 1800 vielleicht eine oder ein paar) »sicher etablierten liberalen « Gesellschaften ungerechte Kriege führten bzw. führen und gegen die Menschenrechte handelten bzw. handeln ${ }^{31}$. Drittens ist zu beachten, dass diese liberalen Gesellschaften in Bündnissen mit outlaw regimes standen, wie z.B. der Sowjetunion. Viertens muss festgehalten werden, dass die Periode, seit der genügend »sicher etablierte liberale Gesellschaften « existieren und entsprechende Konfliktmöglichkeiten zwischen ihnen entstehen könnten, viel zu kurz ist, als dass die Beobachtungen aussagekräftig sein könnten. Die Zeitspanne umfasst nur wenig mehr als ein halbes Jahrhundert und ist darüber hinaus durch besonders günstige geschichtliche Umstände gekennzeichnet - Überfluss an materiellen Gütern in den mächtigen, sicher etablierten liberalen Gesellschaften, gemeinsame Bedrohung durch die sozialistischen Staaten und die entsprechende Notwendigkeit eines Bündnisses innerhalb Europas sowie zwischen Europa und den USA.

Rawls lehnt in seiner völkerrechtlichen Theorie - wie Kant - einen Weltstaat ab. Er spricht sich hingegen (ebenfalls im Sinne Kants) für die Existenz einer Gesellschaft von Staaten aus, die durch gemeinsam festgelegte und im Recht der Völker gegründete Kooperationsbedingungen miteinander verbunden sind. Eine Weltregierung würde »entweder einen weltweiten Despotismus mit sich bringen« oder »über ein fragiles Reich herrschen «, »das durch Völker, die versuchen, ihre politische Freiheit und Autonomie zu gewinnen, zerrissen würde «32. Diese Weltregierung würde zwischen Unterdrückung und Anarchie schwanken, weil die geistigen Inhalte, die die verschiedenen Völker gestalten und die sie voreinander unterscheiden, einen bestimmenden Einfluss haben. Diese Inhalte sind Grund des Strebens nach Unabhängigkeit.

Rawls erweitert sein Modell zum zweiten Mal: Er versucht, es auf die Völker anzuwenden, die er als »achtbare« bezeichnet. Diese sind solche, die zwar nicht gemäß

28 Vgl. I. Kant, Vorarbeiten zur Einleitung in die Rechtslebre. Akademieausgabe, Bd. 23, S. 257.

29 Vgl. RdV, S. 16, 55 f.

30 RdV, S. 58.

31 Z. B. Großbritannien in Irland; die USA in Vietnam und im Irak; beide im Zweiten Weltkrieg gegen Japan und Deutschland, wie selbst Rawls anerkennt (vgl. RdV, S. 120, 124 ff.); Israel in Palästina. Über den letzten Krieg im Irak vgl. D. Blumenwitz, »Die völkerrechtlichen Aspekte des Irak-Konflikts«, in: Zeitschrift für Politik 2003, S. 301334.

32 Vgl. RdV, S. 40 f.; vgl. I. Kant, Zum ewigen Frieden, aaO. (FN 8), S. 360 ff. 
liberal-demokratischen Grundsätzen organisiert sind, die aber das Recht der Völker achten $^{33}$, in denen es Konsultationshierarchien gibt ${ }^{34}$ und bei denen drei weitere Voraussetzungen erfüllt sind: (a) Das Rechtssystem gewährleistet allen Volksmitgliedern das Recht auf Leben (»auf das für die eigene Subsistenz und Sicherheit Nötige«), auf Freiheit (Freiheit von Sklaverei, Leibeigenschaft und Zwangsarbeit; ein hinreichendes Maß an Gewissensfreiheit), auf persönliches Eigentum und auf formale Gleichheit (gleiche Fälle sollen gleich behandelt werden) ${ }^{35}$. (b) Das Rechtssystem muss so beschaffen sein, »dass es allen Personen auf dem Territorium des Volkes moralische bona-fide-Pflichten und Verpflichtungen« auferlegt. Die Mitglieder des Volkes werden als rational, achtbar, verantwortlich und als zur Teilnahme am sozialen Leben befähigt betrachtet. Sie erkennen Pflichten und Verpflichtungen als mit ihrer »Gemeinwohlvorstellung der Gerechtigkeit« übereinstimmend an, nicht als bloß zwangsläufige Befehle. (c) Auf Seiten der Richter und Beamten, die das Rechtssystem verwalten, besteht die nicht unbegründete Überzeugung, dass das Recht tatsächlich von einer Gemeinwohlvorstellung der Gerechtigkeit geleitet wird ${ }^{36}$.

In den achtbaren Völkern gibt es hierarchisch organisierte Gruppen. Die Individuen werden hier nicht als Gleiche anerkannt, aber allen wird Rationalität, Verantwortlichkeit und die Fähigkeit zum moralischen Lernen zugesprochen. Innerhalb der Gruppen haben sie ihr besonderes Handlungsfeld. Abweichende Meinungen werden erlaubt und sogar körperschaftlich repräsentiert. Die Gruppen sind juristisch anerkannt und spielen eine Rolle bei den sozialen und politischen Entscheidungen, besonders mit ihrer Teilnahme an Konsultationsverfahren. Sie bilden eine Art institutionelle Grenze der Macht, beschränken und erhalten die Rechte und Pflichten ihrer Mitglieder ${ }^{37}$.

Achtbare Völker sind wohlgeordnet, und als solche nehmen sie die acht Grundsätze des Rechts der Völker im Urzustand an. Für diese Völker wird der Urzustand nur einmal vorausgesetzt: Nur für die Annahme der Grundsätze des Rechts der Völker. Der erste (»heimische«) Urzustand wird hier nicht vorausgesetzt, denn die achtbaren Völker sind nicht liberal-demokratisch, sondern hierarchisch strukturiert ${ }^{38}$.

Zwischen liberalen und achtbaren Völkern gibt es eine gemeinsame öffentliche Vernunft, die sich auf den Inhalt des Rechts der Völker bezieht. Dieses Recht der Völ-

33 Die achtbaren Gesellschaften verfolgen keine aggressiven Ziele und erkennen an, dass sie die legitimen Ziele auf friedlichem Wege erreichen müssen. Sie können auf umfassenden Lehren gegründet sein und achten die Ordnung anderer Gesellschaften, wenn sie im internationalen Bereich handeln; vgl. RdV, S. 79.

34 Vgl. RdV, S. 87 ff.

35 Die so verstandenen Menschenrechte überwinden nach Rawls ihren politisch provinziellen Charakter und werden universal. Sie müssen nicht (obwohl sie können) durch eine philosophisch oder religiös umfassende Lehre begründet werden; RdV, S. 80.

36 RdV, S. 79-81. Diese Kriterien bestimmen die "Achtbarkeit" als Kategorie, die denen der Vernünftigkeit ähnlich sind, aber wenig umfassender als diese sind; ebd. S. 81 f., 5, 108. Nach Rawls sind andere Arten von achtbaren Völkern vorstellbar, er erwähnt sie aber nicht weiter; ebd. S. 77 f.

37 Vgl. RdV, S. 79, 86 ff., 108.

38 RdV, S. 86. 
ker ist nach Rawls nicht ethnozentrisch oder provinziell, denn es umfasst auch nichtliberale Völker und seine achtbaren Religionen und Vorstellungen. Es erfüllt das Kriterium der Reziprozität: Es verlangt von anderen (nicht-liberalen) Gesellschaften lediglich solche Dinge, die sie vernünftigerweise zugestehen können, ohne sich damit in eine untergebene Position zu begeben. Es verlangt von den achtbaren Gesellschaften nicht, dass sie ihre religiösen Institutionen aufgeben und liberale annehmen ${ }^{39}$.

Rawls stellt sich die Frage nach der Rechtfertigung der Anerkennung von achtbaren Völkern. Wenn solche Völker ihrer Mitglieder nicht als freie und gleiche Individuen behandeln, warum sollen sie dann von den liberal-demokratischen Völker als gleiche Völker behandelt werden? Die Nichtanerkennung ist jedoch eine Ursache für schlechte Beziehungen zwischen Völker. Das kann in extremen Fällen zum Krieg führen. Wenn die achtbaren Völker die grundsätzlichen Rechte der Individuen und Hierarchien sowie das Recht der Völker anerkennen, sind ihre Differenzen mit den liberalen Völkern nicht so ernst, um nicht gute Beziehungen zu ihnen zu pflegen. Wenn ein Volk die Kriterien der Achtbarkeit erfüllt, soll es als Mitglied der Gesellschaft der Völker anerkannt werden ${ }^{40}$.

\section{3. Änderung der nicht-woblgeordneten Gesellschaften gemäß der Grundsätze des Rechts der Völker}

Rawls nennt »nichtideale Theorie« die Betrachtung der Umstände, unter denen auf der Welt Ungerechtigkeit und Übel stattfinden, sowie die Überlegungen im Hinblick auf Möglichkeiten ihrer Überwindung. Teile dieser nichtidealen Theorie sind die Theorie des gerechten Krieges (bei der es um die Beziehungen zwischen wohlgeordneten Gesellschaften und outlaw regimes geht) und die der »belasteten Gesellschaften«.

\section{a) Kriegsvölkerrecht}

Die einzige allgemeine Rechtfertigung des Krieges ist nach Rawls die Selbstverteidigung. Andere Motive seien nicht annehmbar und der Staat, der versucht, andere Ziele durch Krieg zu erreichen, werde zum outlaw regime ${ }^{41}$. Außerdem erkennt er das ius ad bellum auch in Fällen der Verteidigung von alliierten Staaten (die ihrerseits keine outlaw regimes sein dürfen) gegen outlaw regimes an sowie für humanitäre Interventionen in Fällen schwerer Verletzungen der Menschenrechte ${ }^{42}$.

Rawls nennt sechs Regeln, die die Staaten während des Krieges beachten müssen:

1) Ziel des Krieges ist ein gerechter und dauerhafter Frieden zwischen den Völkern

39 Vgl. RdV, S. 153.

40 Vgl. RdV, S. 72 ff., 84 f., 96 ff., 4 f.

41 Vgl. RdV, S. $114 \mathrm{ff}$.

42 Vgl. RdV, S. 97 f.. Wenn sich ein Staat in einer der drei beschriebenen Situationen befindet, hat er das Recht, Krieg zu führen. Dieses Recht aber unterliegt einer Einschränkung: Nur Gesellschaften, die nicht aggressiv sind und die die Menschenrechte achten, haben ein Recht zur Kriegsführung; ebd. 117 
und besonders mit dem gegenwärtigen Feind des jeweiligen kriegsführenden Volkes. 2) Wohlgeordnete Völker führen nur gegen nicht wohlgeordnete Staaten Krieg, die deren Sicherheit und freie Institutionen gefährden und den Krieg verursachen. 3) Es muss sorgfältig zwischen drei Gruppen unterschieden werden: Den Führern und Beamten eines outlaw regimes, seinen Soldaten und seiner Zivilbevölkerung. Die zwei letzteren Gruppen, oft in Unwissenheit gehalten, sind nicht verantwortbar zu machen. 4) Wohlgeordnete Völker müssen aus zwei Gründen so weit wie möglich die Menschenrechte der Mitglieder der anderen Seite, sowohl der Zivilisten als auch der Soldaten, achten: Weil das Recht der Völker es gebietet und weil man auf diese Weise den Inhalt der Menschenrechte am besten vermittelt. 5) Die wohlgeordneten Völker müssen bereits während des Krieges im Voraus zu erkennen geben, welche Art von Frieden und welche Art von Beziehungen sie anstreben. Sie werden im Krieg selbst zeigen, um welche Art von Volk es sich bei ihnen handelt ${ }^{43}$. 6) Das instrumentelle Zweckmitteldenken darf stets nur eine beschränkte Rolle bei der Beurteilung der Angemessenheit einer Handlung oder eines politischen Programms spielen. Diese Art des Denkens muss stets im Rahmen der obigen Grundsätze und Annahmen stattfinden und durch diese streng begrenzt werden ${ }^{44}$.

Es gibt aber eine Ausnahme für diese Begrenzungen: Die Situation des äußersten Notstandes. In diesem Fall könne die strenge Geltung des Status von Zivilisten aufgehoben werden. Unschuldige Zivilisten dürften hier also direkt angegriffen werden, wenn bestimmte Bedingungen erfüllt sind: Es soll sich um einen äußersten Notstand handeln (die Existenz achtbarer oder liberaler Völker muss in Gefahr sein). Die Vorteile des Angriffs sollen nicht zweifelhaft und geringfügig, sondern bedeutend sein. Das angreifende Volk soll über keine anderen Mittel verfügen, um die Macht des anderen Volkes zu brechen ${ }^{45}$.

Rawls setzt seine Lehre der christlichen Lehre entgegen, die direkte Angriffe gegen die Zivilbevölkerung absolut verbietet, weil es sich hier um die Verletzung des Lebens von Unschuldigen handelt. Nach Rawls soll der Staatsmann, d.h. der, der die Regierung führen und einen stabilen Frieden schaffen soll, nur gemäß den Interessen des wohlgeordneten Regimes - und nicht gemäß den Prinzipien der religiösen, moralischen oder philosophischen Lehren, nach denen er lebt - politisch handeln ${ }^{46}$.

Obwohl Rawls den Krieg gemäß bestimmter Kriterien begrenzt, erlaubt sein Recht der Völker den Angriff auf unschuldige Zivilisten des feindlichen Staates. Die Notwendigkeit des Angriffs wird einseitig bestimmt, d.h. wer über Leben und Tod von Unschuldigen entscheidet, ist eine der Seiten im Konflikt. Die Missbrauchsmöglichkeiten sind also nicht abzusehen. Nachdem eine der Parteien im Konflikt das Verbot, Unschuldige zu töten, verletzt hat, ist damit zu rechnen, dass der Gegner auf diese Mittel nicht verzichten wird, um Vergeltung zu üben. Die Risiken einer Eskalation des Konfliktes steigen in dem Maße, wie parallel die eines stabilen zukünftigen Friedens sinken. Die konkreten Folgen der Theorie sind hoch aktuell:

43 Vgl. I. Kant, Zum ewigen Frieden, aaO. (FN 8), S. 346 f.

44 Vgl. RdV, S. 119-121.

45 Vgl. RdV, S. 123 f.

46 Vgl. RdV, S. $129 \mathrm{f}$.

ZfP 52. Jg. 3/2005 
Mitglieder abhängiger Völker - wie Palästinenser und Iraker - wären, wenn sie über keine anderen Mittel verfügen, um ihre freie politische Existenz zu erreichen, zum Mord an Unschuldigen berechtigt.

Die Erlaubnis zur Tötung Unschuldiger missachtet die grundlegendste zwischenmenschliche Pflicht. Es ist sinnlos, ein Recht der Völker vorzuschlagen, d.h. einen Zusammenhang von Normen, die das gerechte Handeln zwischen Menschen bestimmen, das aber gleichzeitig die Möglichkeit der totalen Beseitigung einer menschlichen Handlungsweise, die ungerechte Tat schlechthin, festlegt und rechtfertigt.

Die Frage, wer entscheidet, bleibt im Kriegsvölkerrecht Rawls' unbeantwortet. Welcher Staat ein outlaw regime ist, welches Volk ein achtbares oder liberales Volk, welcher Krieg ein gerechter oder ungerechter Krieg ist, darf letzten Endes von den Konfliktparteien entschieden werden. Bisher hat gegolten, dass es zwischen einzelnen Staaten keine allgemein gültigen juristischen Beurteilungen geben darf, denn sie sind gleich (UN-Charta, Art. 2, 1). Die hier vorgestellte Theorie - mit ihren moralisch-politisch diskriminierenden Unterscheidungen und mit der Rechtfertigung von einseitig qualifizierten Interventionskriegen - gefährdet diesen Grundsatz des Völkerrechts sowie das Gewaltanwendungsverbot der UN-Charta (ebd. Art. 2, 4). Das Recht der Völker Rawls' kann schnell zur Waffe der großen Mächte werden.

\section{b) Theorie der belasteten Gesellschaften}

Belastete Gesellschaften oder Gesellschaften, die unter ungünstigen Umständen leiden, sind solche, die weder expansionistisch noch aggressiv sind, denen es aber an politischen und kulturellen Traditionen, an Humankapital, an Know-How oder an den nötigen materiellen und technologischen Ressourcen mangelt, die notwendig sind, um wohlgeordnet zu sein ${ }^{47}$. Es geht dabei nicht nur um bloße wirtschaftliche Probleme. Deshalb sollen Hilfsmaßnahmen nicht allein wirtschaftlich sein; wirtschaftliche Hilfe ohne die notwendige vorherige Ausbildung zu leisten, kann eine destabilisierende Verschwendung sein. Es handelt sich hauptsächlich um die Gestaltung eines institutionellen Systems für die Erziehung und Ausbildung der Bevölkerung gemäß den Prinzipien und Gewohnheiten einer wohlgeordneten Gesellschaft.

Rawls schließt eine universale Anwendung des Differenzprinzips aus. Die Hilfe soll nur das Erreichen eines akzeptablen Minimums ermöglichen, also einer Situation, in der jeder Mensch die Güter hat, die für ein lebenswertes Leben notwendig sind. Ziel ist, dass die unterstützten Gesellschaften ihre Entscheidungen vernünftig und rational treffen können und somit in die Gemeinschaft der wohlgeordneten Völker aufgenommen werden können ${ }^{48}$. Die Gerechtigkeitsprinzipien der Theorie der Gerechtigkeit werden unter Beachtung eines vermittelnden Faktors, der Völker, angewendet.

47 Vgl. RdV, S. 131.

48 Vgl. RdV, S. 132 ff., 141 ff. Rawls fordert eine Art von zarter Klugheit in der staatlichen Hilfstätigkeit: Es gibt kein fertiges Rezept, aber die Maßnahmen sollen nicht durch Gewalt geleitet werden; man soll die Entstehung einer gewissen Affinität mit der unterstützten Gesellschaft suchen; ihre Autonomie muss dafür geachtet werden; ebd. S. 138 f. 


\section{Schluss}

Die völkerrechtliche Theorie Rawls' ist in mehrerlei Hinsichten im guten Sinne des Wortes realistisch. Sie erkennt nicht-liberale demokratische achtbare Völker an und toleriert den wohlwollenden Absolutismus. Andernfalls drohen Konflikt und Krieg (es ist nur fraglich, ob sich die Anerkennung bzw. Tolerierung mit dem ersten Gleichheitsprinzip der Theorie der Gerechtigkeit von 1971 verträgt). Sie ist auch realistisch, weil sie die Möglichkeit des Krieges anerkennt; diese Anerkennung ist die notwendige Basis für eine Begrenzung des Phänomens. Rawls versucht nicht, weltweite wirtschaftliche und soziale Gleichheit zu schaffen, sondern nur akzeptable Minima zu sichern. Die universale Gleichheit ist schwierig zu realisieren und sie kann ernste Probleme mit sich bringen (Konflikte und Kriege wegen der Verteilungen). Da Rawls dies anerkennt, ist seine Theorie in einem dritten Sinn realistisch. Die Ablehnung einer Weltregierung und die Unterscheidung zwischen Menschenrechten, die für liberale Gesellschaften gültig sind, und grundsätzlichen Menschenrechten, sind auch realistische Elemente der Theorie.

\section{Ein grundlegendes Problem}

Rawls versucht, eine politisch autonome, d.h. nicht auf umfassenden Lehren bzw. ontologischen und anthropologischen Begründungen gestützte Gesellschaftsordnung und ein autonomes Recht der Völker festzulegen ${ }^{49}$. Dies bringt seine Theorie in eine prekäre Situation. Ontologie, Anthropologie und Politik müssen unterschieden werden, aber sie sind nicht in der Weise voneinander zu trennen, dass ein autonomer politischer bzw. juristischer Konsens gefunden werden kann. Eine politische Handlung, die von den konstitutiven Elementen des Seins oder des menschlichen Daseins abzusehen versucht, wird sich nicht realisieren lassen und sie wird den Entfaltungsmöglichkeiten des Menschen schaden ${ }^{50}$. Sofern es kein vom Sein getrenntes Sollen geben kann und das Sein keine rein neutrale Tatsache, sondern sinnhaft ist, ist die qualitative Dimension auch auf politischem bzw. juristischem Gebiet bedeutend und die Entscheidungen müssen sich darauf stützen: Die Ziele des Menschen müssen berücksichtigt werden ${ }^{51}$.

49 Es gibt zu diesem Thema vielfältige Literatur. Von besonderer Bedeutung ist hier die Kritik der so genannten Kommunitaristen. Vgl. dazu: Ch. Taylor, Negative Freiheit. Frankfurt/M. 1988; ders., Philosophy and the Human Sciences. Philosophical Papers 2. Cambridge, New York 1985, S. 187-210; ders., Sources of the Self. The Making of the Modern Identity. Cambridge 1989; A. MacIntyre, Der Verlust der Tugend. Zur moralischen Krise der Gegenwart. Frankfurt/M./New York 1987; M. Sandel, Liberalism and the Limits of Justice. Cambridge 1982.

50 Vgl. Ch. Taylor, Negative Freiheit, ebd., S. 148 ff., 154. Die Menschenrechte z.B. sind ohne eine Anthropologie, die sie erklärt und begründet, unverständlich.

51 Alles, was ist, ist. Außer dem Sein gibt es nichts. Die qualitative Dimension ist also im Sein und kann nicht etwas vom Sein Getrenntes sein. Die Trennung zwischen einer ontologischen und einer als autonom konzipierten normativen Dimension ist zu diskutieren. Das Normative ist nicht vom Sein zu trennen. Das Sein enthält den Sinn, der Sinn ist (obwohl auf eine andere Weise als die wirklichen Dinge). Dieser Sinn des Seins begründet jede Entscheidung: Alles, was der Mensch vorzieht, hat sein Grund in dieser qualitativen Dimension; vgl. H. Kuhn, Das Sein und das Gute. München 1962, S. 26 f., 60 f., 64, 70 f., 77; H. Herrera, Sein und Staat. Die ontologische Begründung der politischen Praxis bei H. Kuhn. Würzburg 2005, S. 25 ss. 
Die Theorien der Praxis und des Politischen sind nicht $»$ politisch autonom «52. Eine Theorie der Gerechtigkeit, die die Gerechtigkeit gemäß äußerer Regel des Zusammenlebens zu bestimmen versucht und die die umfassenden Lehren über das Sein und den Menschen auf das private Gebiet verbannt, wird, wenn sie in einer Gesellschaft umgesetzt wird, zu einem Wechsel der in einer solchen Gesellschaft erlaubten, geförderten, verbotenen und abgelehnten Handlungen führen. Und das ist alles andere als politisch autonom. Die Theorie Rawls' ist in diesem Sinn den umfassenden Lehren ähnlich: Sie versucht, den Einfluss anderer Lehren abzubauen, um bestimmte praktische Ziele zu verwirklichen (im Falle Rawls': Frieden, liberale Prinzipien usw.). Sie tritt dadurch in Konkurrenz mit anderen Lehren und steht notwendig auf einem oberflächlichen Niveau: Weil sie nicht ihre ontologischen und anthropologischen Voraussetzungen reflektiert, neigt sie dazu, dogmatisch zu sein. Sie stützt sich - wie Rawls anerkennt - auf gesetzte, nicht begründete Prinzipien ${ }^{53}$.

Die Priorität der Normen einer formalen - nicht ontologisch bzw. anthropologisch begründeten - Gerechtigkeit vor der ontologischen Auffassung des Guten ${ }^{54}$ schwächt eine wesentliche Voraussetzung des Gerechten, die Freundschaft. Echte Freundschaft entsteht nur, wenn das Gute des Anderen gewollt wird: zum einen als in seiner Existenz gut, zum anderen seine persönliche Entfaltung. Dies setzt die $A n-$ erkennung des Guten im Sein voraus, die Rawls nicht vollzieht - wie auch nicht Kant, für den das Gute letztendlich Sache des Glaubens und nicht des Wissens ist ${ }^{55}$. Ohne diese Anerkennung ist die Begründung von Freundschaft, die fähig ist, Lust, Nützlichkeit und Behagen zu relativieren, nicht möglich und es ist nicht erklärbar, wie Freundschaft diese Relativierung tatsächlich vollzieht. Die Orientierung am Guten des Anderen macht die Freundschaft zu einem notwendigen Ziel: Echte Freundschaft soll immer geachtet werden. Die Freundschaft als sinnhafte Umgangsweise aktualisiert die Motivation, den Anderen und seine Freiheit zu respektieren und begründet die Forderung nach solchem Respekt. Das Recht lässt sich ohne Freundschaft nicht verwirklichen wegen des Mangels an Motivation und Rechtfertigung. Freundschaft ist insofern Voraussetzung für Recht. Ohne Freundschaft verliert sich eine Beziehungsform, die Teilnahme am Sinn begünstigt. Die Teilnahme isolierter Individuen am Sinn erzeugt ein Problem gegenüber Dritten, wenn die Individuen durch ihre Versuche, am Sinn teilzunehmen, die Bewegungsmöglichkeiten der Dritten und deren Teilnahme am Sinn behindern bzw. erschweren, was zwischen begrenzten Menschen geschehen muss. In einer Gesellschaft ohne Freundschaft sind die Individuen wenig dazu geneigt, Pflichten zugunsten anderer zu er-

52 Vgl. W. Wieland, »Praktische Philosophie und Wissenschaft«, in: M. Riedel (Hg), Rehabilitierung der praktischen Philosophie. Freiburg 1972, Bd. 1, S. 512 ff., 530 ff.; H. Kuhn, »Praxis und Theorie im platonischen Denkens", in: P. Engelhardt (Hg.), Zur Theorie der Praxis. Interpretation und Aspekte. Mainz 1970, S. 41; E. Voegelin, Rasse und Staat. Tübingen 1933, S. 4; Aristoteles, Nikomachische Ethik 1094a 1-7.

53 Vgl. TdG, S. 38 f., RdV, S. 108.

54 Vgl. TdG, S. 434.

55 I. Kant, Kritik der praktischen Vernunft, Akademieausgabe, Bd. 5, 122; ders., Die Religion innerbalb der Grenzen der bloßen Vernunft, Akademieausgabe, Bd. 6, S. 6; ders., Kritik der reinen Vernunft, A 810/B 838-A 819/B 847, A 828/B 856-A 829/B 857. 
füllen, mit denen sie nur durch ein formal äußerlich geregeltes Zusammensein verbunden sind ${ }^{56}$. Die Möglichkeiten sinnvollen Lebens sowie Recht und Freiheit werden in einer Gesellschaft von Teufeln (Kant) ernstlich erschwert. Hier ist zu fragen, ob es gerechtfertigt ist, die formalen juristischen Regeln einer solchen Gesellschaft zu beachten.

Die Fähigkeit zur Freundschaft kann nur im Rahmen innerlicher zwischenmenschlicher Beziehungen erreicht werden. Diese notwendige Innerlichkeit wird am besten in gemeinschaftlichen Bereichen ermöglicht. Viel besser z.B. in einer Familie als in einer Gesellschaft, die nur durch äußere, potenziell zwangsläufige, formale Verkehrsregelungen bestimmt ist wie die politische Einheit im Sinne Rawls. Die gemeinschaftlichen Bereiche sind wenig stabil, weil der Mensch begrenzt ist (er kann anderen schaden, konkrete Güter verabsolutieren). Schutz und Förderung sind also bedeutend. Das Verständnis von Recht als äußerliche Regel, die Behauptung der Priorität des formal Gerechten und der autonomen Politik über die Gemeinschaften und ihre eigentümlichen Strukturen und Elemente erschwert dieser Unterstützung und die Entstehung von freundschaftlichen Beziehungen ${ }^{57}$.

Die Autonomie der Theorie Rawls' bezüglich der Ontologie und der Anthropologie entzieht ihr jeden Grund, von dem her sich die Beachtung des Menschen und seiner Möglichkeiten, Sinn zu suchen und zu verwirklichen, rechtfertigen lässt; und so kann Rawls z.B. eine politische bzw. juristische Ordnung ethisch begründen wollen, die gerade die Tötung von unschuldigen Zivilisten rechtfertigt. Ein solcher autonomer Charakter ermöglicht es Rawls ebenso, sich einen ideal konzipierten Begriff von Volk vorzustellen, der von der Struktur des Menschen und dessen Aggressivität getrennt bleibt.

$\mathrm{Da}$ die Theorie Rawls' ontologisch und anthropologisch gesehen unkritisch bleibt, muss dieser sich auf äußerliche - d.h. nicht argumentative - Voraussetzungen stützen, die er nicht reflektiert. Sein Recht der Völker und sein Frieden setzten neben einer Situation des Überflusses an materiellen Gütern (die Theorie - ihr Frieden - versagt im Fall eines absoluten Mangels, der die ganze Welt betrifft ${ }^{58}$ ) die militärische und wirtschaftliche Überlegenheit der liberalen und achtbaren Völker als Gesamtheit voraus. Deshalb kann Rawls politisch »autonome« Grundsätze und Normen einfach vorschlagen, ohne sich auf andere Begründungen als auf die Bezeichnung solcher Prinzipien und Normen als »vernünftig«, »rational« oder »acht-

56 »Freundschaft ist es, die die Staaten erhält«; Nikomachische Ethik 1155a 23-25; vgl. 27 f.; 1155b 17-34; 1160a 18-30; Platon, Politeia, 351c-d; Euthyphron, 7 c-d.

57 Gemeinschaftliche Bereiche wie die Familie sind vor dem staatlichen Recht konstituiert und haben eigene ursprüngliche Begriffe und Strukturen. Das Zusammenleben ihrer Mitglieder kann »weder im Funktionalismus vorherbestimmter Gesetze, noch in Vertragsregelungen aufgelöst werden «; vgl. C. Schmitt, Über die drei Arten des rechtswissenschaftlichen Denkens, Berlin 1993 (Hamburg ${ }^{1} 1934$ ), S. 17. Die staatlich-juristische Verkennung ihrer Elemente, ihre Regelung gemäß allgemeinen Normen zerstört ihr spezifisch rechtliches Wesen und schwächt sie sowie die Möglichkeiten einer sinnvollen persönlichen und politischen Existenz; vgl. H. Herrera, Sein und Staat, aaO. (FN 51), S. 83-95; Ch. Taylor, Negative Freibeit, aaO. (FN 49), S. 154 f., 273. Ebd. S. 146. 
bar « zu stützen, was nicht mehr als »tatsächlich akzeptabel für die Individuen der ideal konzipierten liberalen bzw. achtbaren Völker « bedeutet. Wenn die Macht hingegen auf der Seite der nicht »wohlgeordneten « Gesellschaften wäre, würde die Theorie als ungenügend erscheinen, und es wäre eine gänzlich differente Art von Argumenten nötig (sie müssten sich auf Ontologie und Anthropologie beziehen), um die Herrscher und Untertanen solcher Staaten zu überzeugen.

\section{Zusammenfassung}

In seinem letzten Buch Das Recht der Völker versucht Rawls, einen gerechten Weltfrieden auf bestimmte Prinzipien zu gründen, die die »liberal-demokratischen « und "achtbaren "Völker in einer vorausgesetzten, ursprünglichen Situation annehmen sollten. Diese ursprüngliche Situation zeigt Ähnlichkeiten mit dem in der Theorie der Gerechtigkeit von 1971 beschriebenen Urzustand (so z. B. wird in beiden ein so genannter "Schleier des Nichtswissen" vorausgesetzt, der verhindern soll, dass die Beteiligten ihre Position bezüglich der anderen kennen). Trotzdem fügt Rawls Änderungen hinzu. Die wichtigste ist, dass der Ausgangspunkt seiner völkerrechtlichen Theorie nicht mehr Individuen, sondern Völker sind. Die Grundsätze, auf denen Rawls den Frieden zu stützen versucht, sind unter vielen Aspekten plausibel, unter anderen zeigen sie aber bestimmte Schwächen, die teilweise auf die Theorie von 1971 zurückzuführen sind.

\section{Summary}

This article analyzes the last book that J. Rawls published in his lifetime: The Law of Peoples. Rawls' intention is to lay the foundations of world peace based on certain principles, which should be acceptable to both liberal democratic and organized nations in a supposed originary situation, described by the author. This supposed originary situation resembles that supposed originary situation stated by Rawls in Theory of Justice, 1971 (i.e. they both assume the existence of a sort of veil of ignorance that enables the counterparts of an agreement to know each other's position). Nevertheless, some adjustments are made. The main one is that it is no longer the initiative of individuals but of the nations themselves. In spite of certain weaknesses which the article attempts to identify, and whose antecedents had already been recorded in the 1971 theory, the principles on which Rawls intends to establish world peace are, in many aspects, plausible. 